\title{
Minimal important improvement thresholds for the six-minute walk test in a knee arthroplasty cohort: triangulation of anchor- and distribution-based methods
}

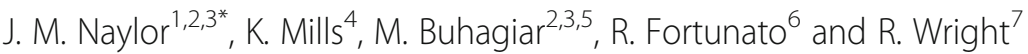

\begin{abstract}
Background: The 6-minute walk test (6MWT) is a commonly used metric for measuring change in mobility after knee arthroplasty, however, what is considered an improvement after surgery has not been defined. The determination of important change in an outcome assessment tool is controversial and may require more than one approach. This study, nested within a combined randomised and observational trial, aimed to define a minimal important improvement threshold for the 6MWT in a knee arthroplasty cohort through a triangulation of methods including patient-perceived anchor-based thresholds and distribution-based thresholds.

Methods: Individuals with osteoarthritis performed a 6MWT pre-arthroplasty then at 10 and 26 weeks post-surgery. Each rated their perceived improvement in mobility post-surgery on a 7-point transition scale anchored from "much better" to "much worse". Based on these responses the cohort was dichotomised into 'improved' and 'not improved'. The thresholds for patient-perceived improvements were then identified using two receiver operating curve methods producing sensitivity and specificity indices. Distribution-based change thresholds were determined using two methods utilising effect size (ES). Agreement between the anchor- and distribution-based methods was assessed using kappa.
\end{abstract}

Results: One hundred fifty-eight from 166 participants in the randomised cohort and 222 from 243 in the combined randomised and observational cohort were included at 10 and 26 weeks, respectively. The slightly or more patient-perceived improvement threshold at 26 weeks (an absolute improvement of $26 \mathrm{~m}$ ) was the only one to demonstrate sensitivity and specificity results both better than chance. At 10- and 26-weeks, the ES based on the mean change score divided by the baseline standard deviation (SD), was an absolute change of 24.5 and $37.9 \mathrm{~m}$, respectively. The threshold based on a moderate ES (a 0.5 SD of the baseline score) was a change of 55.0 and $55.4 \mathrm{~m}$ at 10- and 26-weeks, respectively. The level of agreement between the 26-week anchor-based and distribution-based minimal absolute changes was very good ( $k=0.88$ (95\% Cl 0.810 .95$)$ ).

Conclusion: A valid threshold of improvement for the $6 \mathrm{MWT}$ can only be proposed for changes identified from baseline to 26 weeks post-surgery. The level of agreement between anchor- and distribution-based methods indicates that a true minimal or more threshold of meaningful improvement following surgery is likely within the ranges proposed by the triangulation of all four methods, that is, 26 to $55 \mathrm{~m}$.

Keywords: Arthroplasty, knee, Arthroplasty, Six-minute walk test, Mobility test, Clinimetric

\footnotetext{
* Correspondence: Justine.Naylor@sswahs.nsw.gov.au

${ }^{1}$ Whitlam Orthopaedic Research Centre, Orthopaedic Department, Liverpool

Hospital, Locked Bag 7103, Liverpool BC, 1871 Sydney, NSW, Australia

${ }^{2}$ South West Sydney Clinical School, UNSW, Sydney, Australia

Full list of author information is available at the end of the article
} 


\section{Background}

The 6-min walk test (6MWT) is a simple, objectively measured, physical test that is used to evaluate improvement in functional ambulation after TKA [1-8]. Simply stated, it is a test conducted in- or out-side on level ground where the participant is required to walk laps of a 25 or $30 \mathrm{~m}$ track [9]. Participants and observers are given standardised instructions on how to perform the test, and the distance walked over the 6-min period independent of rest periods is recorded. The use of the test in the TKA population arguably has content (face) validity as improvement in mobility is regarded as a primary goal of surgery [10] and rehabilitation after TKA surgery [11]. Further, construct validity for the test (that is, that the test is actually a measure of functional ambulation) for this population is derived from evidence that performance in the $6 \mathrm{MWT}$ has been shown to be an excellent predictor of performance in a more arduous 30-min walk test [11]. The test-retest reproducibility of the 6MWT is also excellent in TKA recipients [12] as well as in people with osteoarthritis awaiting arthroplasty $[8,13]$, and the test is highly responsive [8], indicating the test has the ability to detect change [14].

Interestingly though, despite demonstrating sound clinimetric properties and despite its common use both in the clinic $[13,15,16]$ and in clinical trials $[1-8]$, there are no published data on what may be minimal, moderate or large improvements in this test as perceived by the patient following TKA. Knowledge of what are considered small or large changes by the patient may be relevant for determining whether or not a change in therapy is indicated (at the level of the individual) as well as for sample size calculations for clinical trials [17-19]. Data exist on what minimal important differences (MID) are detectable for this test in this population using distribution-based methods based on observed scores $[8,20]$. These methods express change in terms of a standardised metric such as 0.5 of a standard deviation (0.5SD) or the standardised error of measurement (SEM) [14, 21]. The SEM has been reported to be $28.5 \mathrm{~m}$ in people with knee osteoarthritis awaiting TKA [13]. Similar values have been reported six (25.5 m [12]) and eight weeks (26 m, [8]) post-TKA. Mizner et al [20] report the ES to be $0.66(81 \mathrm{~m}) 1$ year after surgery. However, distributional methods are criticised for ignoring the clinical importance of the magnitude of the change, for not including a measure of change as perceived by the patient, and for not necessarily being a 'minimal' change [14, 20]. An alternative method for determining MIDs, which does incorporate the views of the patient, is an anchor-based method. Anchor-based methods use an external reference (or anchor) by which to categorise respondents $[14,21]$. Often these are patient-based and require the patient to qualify their global perception of change on a transition scale. Criticisms of anchor-based methods, however, are that they are prone to recall bias - that is, faulty recollection by the respondent $[14,21,22]$ - and response-shift - a change in the respondent's understanding of the construct being examined over time [22].

In light of the limitations of the methods to determine minimal or even moderate or large change thresholds, the use of multiple methods and triangulation of methodologies have been recommended [14, 21]. This study aimed, therefore, to define an improvement threshold for the $6 \mathrm{MWT}$ in a TKA cohort through a triangulation of methods using patient-perceived anchor-based improvement thresholds as well as distribution-based improvement thresholds.

\section{Methods}

\section{Study design and setting}

This study was nested within a multicentre, two-armed randomised controlled trial (HIHO) with a third nonrandomised, observational arm [2] (http://clinicaltrials.gov ref NCT01583153). The controlled trial was designed to test the superiority of 10 days of inpatient rehabilitation together with a monitored home program on measured mobility over a monitored home program (usual care) alone following TKA. Those in the observational cohort received the same home program after their TKA. All participants provided informed, written consent and the study was approved by the human research ethics committees of the institutions involved. The protocol for the clinical trial is described in detail elsewhere [2]; a summary of the study procedures is provided herein.

\section{Participant screening and recruitment}

Potential participants were screened by research personnel during their pre-admission visit approximately 4 weeks prior to surgery. Adults presenting to either of two metropolitan hospitals for a primary, unilateral TKA, with a primary diagnosis of knee osteoarthritis were eligible to participate in the RCT. People who were eligible, but declined to be included in the randomised arms of the study, were invited to participate in the observational arm whereby they received usual care. Socio-demographic and anthropometric data were obtained at this time. People who were unable to comprehend the study protocol, unable to perform exercises in an unsupervised environment, unable to attend one of three physiotherapy departments involved in the study, or who had a predisposition to be discharged to a rehabilitation facility (for example, they lived alone), were excluded from the study.

\section{Outcomes and testing procedures}

After consent was obtained, each participant completed patient-reported surveys relevant to the larger study and 
completed a 6MWT on an outside $30 \mathrm{~m}$ straight track according to recommended testing procedures [9]. A practise 6MWT was not undertaken as all patients presenting for TKA at the study hospitals were required to perform the test at several time points whilst awaiting surgery as part of a waitlist management program $[13,15,16]$. At 10 weeks (randomised participants only) and 26 weeks (all participants) post-surgery, the $6 \mathrm{MWT}$ was repeated. Prior to testing, participants were asked to rate their perceived improvement in their mobility three ways; at 10 weeks, anchored to pre-surgery, then at 26 weeks, anchored to both presurgery and 10 weeks.

For rating patient global impression of improvement, we used an anchor-based method commonly recommended for determining the minimal important improvement $[18,23-25]$. Participants were asked to rate their perceived improvement in mobility on a 7-point Likert scale. Each denoted whether they were 'much worse', 'moderately worse', 'slightly worse', 'no change/ same', 'slightly better', 'moderately better', 'much better', compared to how they were prior to surgery. The global style of questioning used - 'How does your walking compare to before surgery?'- was consistent with previous studies which have identified minimum thresholds for improvement for the 6MWT in other clinical populations $[18,19,24]$.

\section{Preliminary analyses}

Prior to analyses of the improvement thresholds, growth curve analyses were conducted to determine whether there were differences in the magnitude (model 1) and rate of change (model 2) in the 6MWT over the followup periods [26, 27]. These analyses allowed us to robustly deal with the change in sample size across different time points, but also indicated whether any improvement thresholds identified could apply across all time periods. The latter was important as MIDs are thought to be time-specific [14, 28, 29]. A third model was fitted to determine the influence of readily measurable patient variables on baseline 6MWT distance and/ or the magnitude and rate of change over time (body mass index (BMI), age, gender, comorbidity count, baseline disease severity). This analysis was necessary as it would identify whether an improvement threshold could apply regardless of participant characteristics. For the purposes of this predictor model, rehabilitation group allocation was ignored as it was found to not significantly interact with baseline 6MWT or improvement in distance over time. To ensure best fit of the data, all models were fitted using an unstructured covariance structure, which requires no assumption in error structure [26].

\section{Analyses of thresholds}

Anchor- and distribution-based approaches were utilised for determining meaningfulness of the improvement thresholds.

For the anchor-based method, identification of the thresholds and determining their acceptability were performed over three stages. Firstly, correlation between the absolute change scores from baseline to each follow-up period and the Likert scale was assessed using Spearman's rank correlation. This was repeated for the relative change scores, where 6MWT distance was expressed as a percentage of baseline. While the optimal correlation coefficient for a typical MID analysis is conventionally regarded as $>0.3$ [14], due to the exploratory nature of this study, we chose to investigate the improvement thresholds that had any statistically significant $(p<0.05)$ correlation.

Secondly, the improvement thresholds were investigated by dichotomising all participants into "improved" and "not-improved" groups. For the minimal group, the dichotomy was set with those reporting slightly improved or more (that is they reported slight, moderate or much better improvement) as the improved group and those reporting no change or worse as the not-improved group. The moderate group split occurred at the moderately better or more level, and the much better difference group only included those reporting they were much better. A priori, we had planned to identify the slight, moderate or much better thresholds in non-overlapping (independent) groups, however, too few people reported to be slightly better or even moderately better. Any conclusive analysis using these original categorisations was precluded, therefore, because such a small sample in the 'slightly better' group threatened the precision of the estimates obtained [30].

Thirdly, the 6MWT data, now dichotomised into those who had reported improvement or not, were plotted on a receiver operating characteristic (ROC) curve, with the improved group as the reference group on all occasions. This was done for all three improvement threshold groups. The area under the curve (AUC) and $95 \%$ confidence intervals $(\mathrm{CI})$ were calculated for each ROC curve in order to provide insight into the discriminatory power of the transition question. These were compared using DeLong's statistic (D) to determine if using the slight, moderate or much better change was a more appropriate method for determining what would be useful clinically or scientifically. An AUC of $75 \%$ or more has previously been proposed to be clinically useful [31]. The threshold of difference was then set using two methods: the first, the top left hand corner of the graph that results in the optimal combination of sensitivity and specificity, known as Youden's method [32]; the second, the $80 \%$ specificity method [33], selects the threshold that has a minimum 
of $80 \%$ specificity while obtaining the highest possible sensitivity. Confidences intervals (CIs) for the sensitivity and specificity of each threshold were calculated using 500 bootstrap samples. Values greater than 50 indicated that the thresholds were better at identifying individuals who would (sensitivity) and would not (specificity) improve to a patient-perceived amount. ROC curves were calculated for the change in 6MWT both in absolute terms and as a percentage of the patient's baseline value.

The distribution-based approach utilised the ES. There are two methods to this approach. The first examines the mean differences between pre- and post-surgical $6 \mathrm{MWT}$ distances and divides them by the standard deviation (SD) of the pre-surgery distance [34]. The second method is to determine $50 \%$ of the SD of the baseline score, which correlates to a moderate effect [35]. This is a commonly used method to obtain a MID [36] and is based on a systematic review of 29 investigations across several disease conditions, which reported that the ES converged on 0.5 SD [35]. These methods were applied to both absolute and relative scores at 10- and 26-weeks post TKA. To examine the concordance in classifications between the anchor- and distribution-based MID thresholds, we used the kappa index of agreement [37]. To obtain $95 \%$ confidence intervals for the kappas, we used 500 bootstrap samples.

\section{Results}

Of the 243 participants included in the larger study, 166 and 77 belonged to the RCT and observational arms, respectively; 158 were available at the 10-week assessment (RCT participants only) and 222 were available at the 26-week assessment (RCT and observational combined). Table 1 summarises the characteristics of

Table 1 Cohort characteristics

\begin{tabular}{llll}
\hline & $\begin{array}{l}\text { Randomised } \\
\text { cohort } \\
n=166\end{array}$ & $\begin{array}{l}\text { Observational } \\
\text { cohort } \\
n=77\end{array}$ & $\begin{array}{l}\text { Entire } \\
\text { cohort } \\
n=243\end{array}$ \\
\hline Age, yrs, mean (sd) & $67.0(8.3)$ & $66.7(8.7)$ & $66.9(8.4)$ \\
Gender, female, n (\%) & $112(68)$ & $35(45)$ & $147(61)$ \\
Body mass index, mean (sd) & $34.7(7.0)$ & $33.0(7.2)$ & $34.2(7.1)$ \\
$\begin{array}{l}\text { Baseline 6-min walk test, } \\
\text { mean (sd) }\end{array}$ & $319.1(109.1)$ & $329.5(114.0)$ & $322.4(110.6)$ \\
Baseline Oxford Knee Score & $17.0(7.1)$ & $17.6(7.7)$ & $17.2(7.3)$ \\
Comorbidity, yes, n (\%) & $127(77)$ & $53(69)$ & $180(75)$ \\
\multicolumn{1}{c}{$\begin{array}{l}\text { Cardiovascular } \\
\text { Gastrointestinal }\end{array}$} & $116(70)$ & $47(61)$ & $163(67)$ \\
Other lower limb & $53(32)$ & $11(14)$ & $64(26)$ \\
or back & $43(26)$ & $21(27)$ & $64(26)$ \\
Diabetes Mellitus & $38(23)$ & $18(23)$ & $56(23)$ \\
Respiratory & $21(13)$ & $10(13)$ & $31(13)$ \\
\hline
\end{tabular}

Key: Five most common comorbidities shown the cohort according to their study grouping (RCT or observational).

\section{Growth curve analyses}

The unadjusted mean preoperative distance was 322.4 (sd 110.6) $\mathrm{m}$ (Table 1) and the unadjusted distances achieved at 10 and 26 weeks were 375.5 (108.26) and 386.7 (113.2) $\mathrm{m}$, respectively. The rates of improvement in the 6MWT changed significantly over time (refer to Appendix 1: Table 4). From 0 to 10 weeks, the adjusted mean increase in distance was $5.3 \mathrm{~m}$ per week for an average male. This rate slowed to a rate of $0.8 \mathrm{~m}$ improvement per week from weeks 10 to 26 . While age and gender influenced preoperative 6MWT distance, they had no effect on the magnitude or rate of change. These results indicated that any significant thresholds that were identified would apply regardless of differences in the participant characteristics included in the model, but owing to the effect of time on improvement, any proposed threshold would be time-specific.

\section{Anchor-based estimation of improvement thresholds Correlation of the transition scale with measured change} While the global transition scale was significantly correlated with the absolute and relative changes in $6 \mathrm{MWT}$ distance from baseline to 10- and 26-weeks, the correlation coefficients were small (Table 2). Further, there was no correlation between the changes in 6MWT from 10-weeks to 26-weeks and the transition scale. As such, the determination of improvement thresholds in the period between the 10- and 26-week follow-ups was excluded from further analysis.

\section{Categorisation of improved versus not improved participants}

At the 10-week assessment, there were 140 (89\%), 128 (81\%) and $85(54 \%)$ people included in the slightly or more, moderate or more and much better improvement categories, respectively. At 26 weeks, there were 188 (85\%), 179 (81\%) and 143 (64\%) in each of the threshold categories. Figure 1 indicates the mean deterioration or improvement in $6 \mathrm{MWT}$ distance observed between categories is not linear; in other words, there is not a graduated increase or decrease from category to category. Through reference to the wide range of maximum negative and positive change observed within each category (Table 3), it can be seen that some people in the 'slightly improved or more' category (that is, they reported they were slightly better or more), demonstrated greater improvement or deterioration than people in the 'much better' category (that is, those who reported they were much better). 
Table 2 Spearman's correlation coefficient between the transition scale, and absolute and relative change in 6MWT distance

\begin{tabular}{|c|c|c|c|}
\hline \multirow[t]{2}{*}{ Change in $6 \mathrm{MWT}$} & \multicolumn{3}{|c|}{ Rating on transition scale } \\
\hline & Baseline to 10-weeks & Baseline to 26-weeks & 10-weeks to 26-weeks \\
\hline \multirow[t]{2}{*}{ Absolute Baseline to 10-weeks } & $0.297(p<0.001)$ & NA & NA \\
\hline & $n=156$ & & \\
\hline \multirow[t]{2}{*}{ Relative Baseline to 10-weeks } & $0.259(p<0.001)$ & NA & NA \\
\hline & $n=156$ & & \\
\hline \multirow[t]{2}{*}{ Absolute Baseline to 26-weeks } & NA & $0.259(p<0.001)$ & NA \\
\hline & & $n=211$ & \\
\hline \multirow[t]{2}{*}{ Relative Baseline to 26-weeks } & NA & $0.239(p<0.001)$ & NA \\
\hline & & $n=211$ & \\
\hline \multirow[t]{2}{*}{ Absolute10-weeks to 26-weeks } & NA & NA & $0.03(p=0.73)$ \\
\hline & & & $n=139$ \\
\hline \multirow[t]{2}{*}{ Relative 10-weeks to 26-weeks } & NA & NA & $0.054(p=0.526)$ \\
\hline & & & $n=139$ \\
\hline
\end{tabular}

NA not applicable

\section{Area under the curve, specificity and sensitivity analyses}

The AUCs indicated that the improvement thresholds were not highly discriminatory with respect to measured changes in the 6MWT (60-75\%) regardless of whether relative or absolute change was used (Table 3). Further, there was no difference in discriminatory power between slight or more, moderate or more and much better definitions of improvement (Fig. 2). The Youden's and $80 \%$ specificity method resulted in different thresholds of slight or more, moderate or more and much better important change. Slight improvement or more at 26-weeks was the only set of thresholds where the specificity and sensitivity were both greater than $50 \%$ for absolute and relative change in both the Youden and $80 \%$ specificity methods. That is, they were the only thresholds considered to have a sensitivity and specificity which were uniformly better than chance, regardless of the analysis method. The absolute values indicated that a "slight improvement or more" ranged from 26 to $64.5 \mathrm{~m}$ improvement in distance, or a relative increase between 11.3 and $18.3 \%$. For the remaining 10- and 26-week thresholds either the sensitivity or specificity were poor suggesting that they are suboptimal for identifying clinically useful improvement in a cohort regardless of the improvement category used (Table 3).

Distribution-based estimation of improvement thresholds At 10-weeks, the ES based on the mean change score $(52 \mathrm{~m})$ divided by the baseline SD $(110 \mathrm{~m})$ was 0.5 . This equivocated to an improvement of $24.5 \mathrm{~m}$ or $12.7 \%$

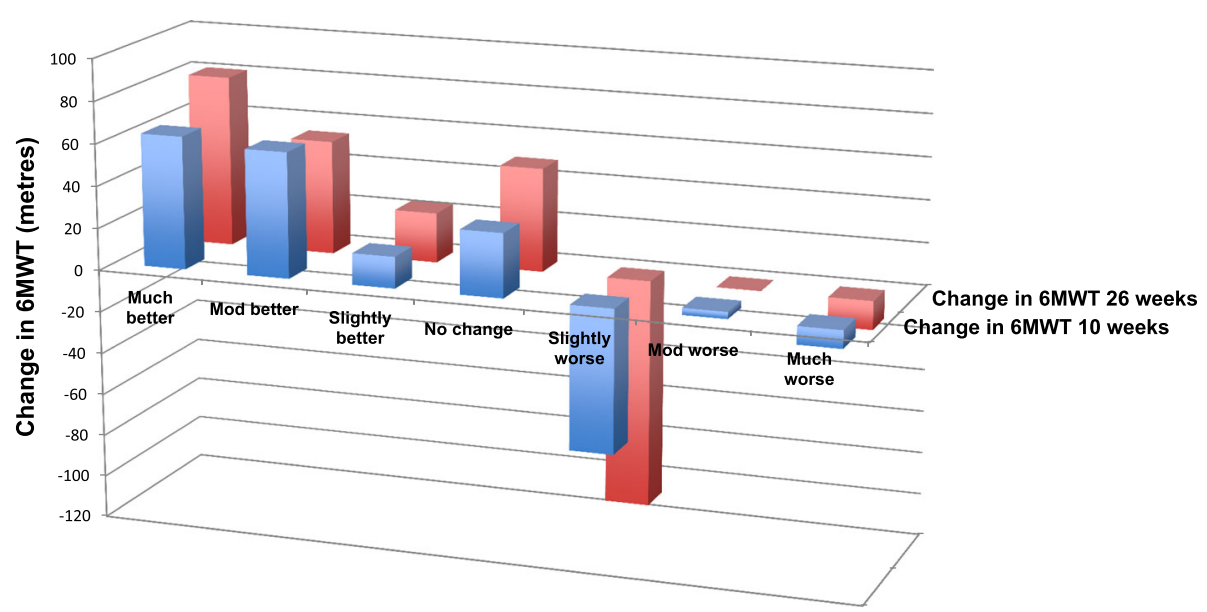

Fig. 1 Mean change in 6MWT distance for each category of the transition scale for baseline to 10-weeks post op (foreground) and baseline to 26-weeks (distance). Sample size varied greatly for each category. At 10-weeks, much better $n=85$, moderately better $n=43$, slightly better $n=12$, no change $n=6$, slightly worse $n=2$, moderately worse $n=7$ and much worse $n=2$. At 26-weeks, much better $n=143$, moderately better $n=36$, slightly better $n=9$, no change $n=9$, slightly worse $n=4$, moderately worse $n=3$, much worse $n=7$ 
Table 3 Area under the curve for ROC curves as well as slight or more, moderate or more and much better important difference for both the absolute and relative changes for the $6 \mathrm{MWT}$

\begin{tabular}{|c|c|c|c|c|c|c|c|c|}
\hline Important change & $\begin{array}{l}\text { Max negative and positive } \\
\text { changes in distance }^{\mathrm{a}}\end{array}$ & AUC (95 \% Cl) & $\begin{array}{l}\text { Youden's } \\
\text { method }\end{array}$ & $\begin{array}{l}\text { Sensitivity } \\
(95 \% \text { Cl) }\end{array}$ & $\begin{array}{l}\text { Specificity } \\
(95 \% \mathrm{Cl})^{b}\end{array}$ & $\begin{array}{l}80 \% \text { Specificity } \\
\text { method }\end{array}$ & $\begin{array}{l}\text { Sensitivity } \\
(95 \% \text { CI) }\end{array}$ & $\begin{array}{l}\text { Specificity } \\
(95 \% \mathrm{Cl})^{b}\end{array}$ \\
\hline \multicolumn{9}{|l|}{10 weeks Absolute } \\
\hline Slight or more $(n=140)$ & $-148.0 \mathrm{~m}$ to $290.0 \mathrm{~m}$ & $70.1 \%(55.58,84.7)$ & $11.5 \mathrm{~m}$ & $70.2(62.41,78.39)$ & $66.7(40,86.67)$ & $53.0 \mathrm{~m}$ & $48.9(41.13,57.45)$ & $80.0(60,100)$ \\
\hline Moderate or more $(n=128)$ & $-148.0 \mathrm{~m}$ to $290.0 \mathrm{~m}$ & $72.3 \%(51.15,83.35)$ & $11.5 \mathrm{~m}$ & $74.4(66.67,82.95)$ & $70.4(51.85,85.19)$ & $53.0 \mathrm{~m}$ & $51.9(42.64,60.47)$ & $81.4(66.67,92.59)$ \\
\hline Much better $(n=85)$ & $-148.0 \mathrm{~m}$ to $252.0 \mathrm{~m}$ & $62.8 \%(53.85,71.78)$ & $11.5 \mathrm{~m}$ & $79.1(70.93,87.21)$ & $48.6(36.39,60)$ & $106.5 \mathrm{~m}$ & $29.1(19.16,38.37)$ & $80.0(71.43,88.57)$ \\
\hline \multicolumn{9}{|l|}{10 weeks Relative } \\
\hline Slight or more $(n=140)$ & -92.6 to $486.0 \%$ & $67.3 \%(51.21,83.44)$ & $5.1 \%$ & $66.7(58.16,74.13)$ & $66.7(40,86.67)$ & $15.1 \%$ & $49.7(40.43,58.16)$ & $80.0(60,100)$ \\
\hline Moderate or more $(n=128)$ & -92.6 to $486.0 \%$ & $70.7 \%(59.05,82.32)$ & $5.1 \%$ & $70.5(62.02,78.29)$ & $70.4(51.85,85.19)$ & $15.5 \%$ & $48.8(40.31,56.59)$ & $81.5(66.67,94.54)$ \\
\hline Much better $(n=85)$ & -92.6 to $486.0 \%$ & $60.7 \%(51.53,69.9)$ & $5.7 \%$ & $54.3(44.29,65.71)$ & $70.5(63.95,83.17)$ & $49.1 \%$ & $23.3(15.12,32.56)$ & $80.0(70.68,88.57)$ \\
\hline \multicolumn{9}{|l|}{26 weeks Absolute } \\
\hline Slight or more $(n=188)$ & $-242.3 m$ to $370.0 m$ & $72.4 \%(62.05,82.7)$ & $26 \mathrm{~m}$ & $72.9(66.21,79.26)$ & $65.2(45.54,82.61)$ & $64.5 \mathrm{~m}$ & $50.0(43.09,56.91)$ & $82.6(67.28,95.65)$ \\
\hline Moderate or more $(n=179)$ & $-242.3 \mathrm{~m}$ to $370.0 \mathrm{~m}$ & $70.6 \%(60.33,80.94)$ & $6.5 \mathrm{~m}$ & $81.6(75.98,87.15)$ & $56.3(38.98,71.88)$ & $71.0 \mathrm{~m}$ & $48.0(41.05,55.31)$ & $81.3(67.28,95.65)$ \\
\hline Much better $(n=143)$ & $-194.0 \mathrm{~m}$ to $370.0 \mathrm{~m}$ & $64.7 \%(56.59,72.77)$ & $8.5 \mathrm{~m}$ & $84.4(78.01,90.07)$ & $41.4(31.43,54.29)$ & $110.5 \mathrm{~m}$ & $35.5(27.66,43.63)$ & $81.4(72.86,90)$ \\
\hline \multicolumn{9}{|l|}{26 weeks Relative } \\
\hline Slight or more $(n=188)$ & -93 to $515.2 \%$ & $71.7 \%(60.64,82.79)$ & $11.3 \%$ & $65.4(58.51,72.09)$ & $73.9(56.52,89.24)$ & $18.3 \%$ & $52.1(45.74,59.04)$ & $82.6(67.28,95.65)$ \\
\hline Moderate or more $(n=179)$ & -93.2 to $515.2 \%$ & $69.6 \%(58.74,80.4)$ & $1.5 \%$ & $82.1(76.54,87.71)$ & $56.3(37.5,71.88)$ & $27.9 \%$ & $40.78(33.78,48.6)$ & $81.3(65.62,93.75)$ \\
\hline Much better $(n=143)$ & -91.3 to $515.2 \%$ & $63.4 \%(55.07,71.78)$ & $1.54 \%$ & $85.1(79.06,90.78)$ & $41.4(30,52.86)$ & $61.0 \%$ & $21.3(14.18,27.66)$ & $84.3(74.29,84.29)$ \\
\hline
\end{tabular}

${ }^{\mathrm{a} C h a n g e}$ in distance from pre-operative $6 \mathrm{MWT}$. Negative integers and percentages $<100 \%$ indicate $6 \mathrm{MWT}$ is less than preoperative values. Positive integers and percentages $>100 \%$ indicate farther distance

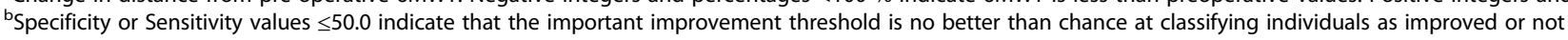




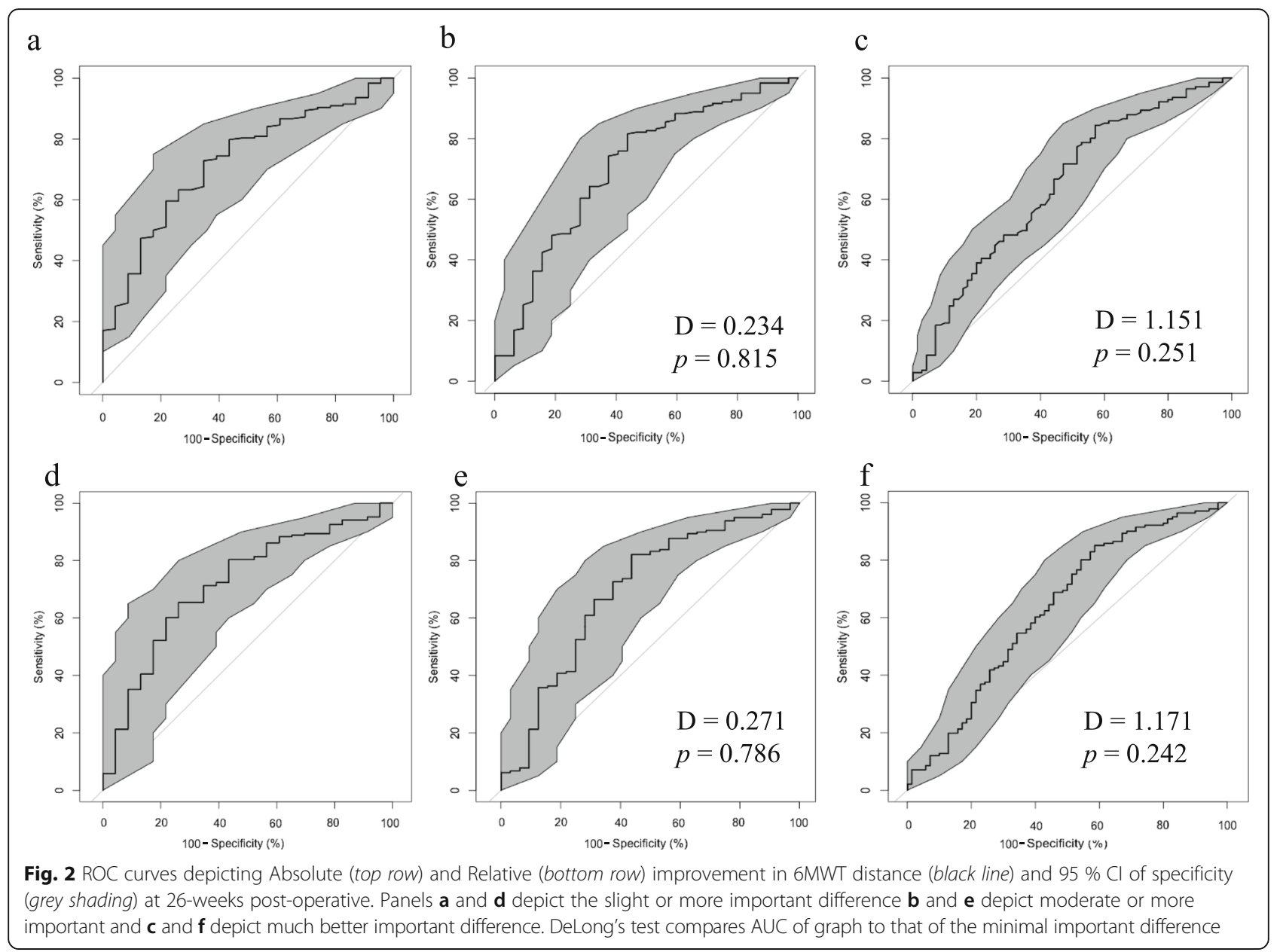

being considered an important change. At 26-weeks this method $(64.8 \mathrm{~m} / 110.8)$ resulted in an ES of 0.6 and proposed threshold of $37.9 \mathrm{~m}$ or $19.6 \%$ change. The distribution MID based on 0.5SD of baseline scores was an improvement of $55.0 \mathrm{~m}$ or $14.6 \%$ of baseline scores at 10 -weeks post-surgery and $55.4 \mathrm{~m}$ or $14.3 \%$ at 26 -weeks post-surgery.

\section{Agreement between anchor- and distribution-based methods}

The kappa level of agreement between the 26-week anchor- and distribution-based minimal change ranged from moderate to strong for absolute change. Agreement between the $80 \%$ specificity ROC method and ES distribution approach exhibited the lowest agreement $(\mathrm{k}=0.67(95 \% \mathrm{CI} 0.57,0.76))$ and the highest agreement occurred between the Youden ROC method and ES distribution approach $(\mathrm{k}=0.88(0.81$, 0.95)). Similarly, when thresholds of relative change were examined, agreement between anchor- and distribution-based approaches ranged from moderate to almost perfect. The lowest agreement was between the Youden ROC method and ES distribution approach $(k=0.69(0.6,0.78))$ and highest was between the Youden ROC method and 0.5SD distribution approach $(\mathrm{k}=0.91(0.85,0.96))$.

\section{Discussion}

To our knowledge, this is the first study to attempt to explore the possibility that patient-perceived improvement thresholds exist for the 6MWT in a TKA cohort. Specifically, we have explored improvement and change thresholds for the 6MWT, using multiple analytical approaches and at two clinically relevant time periods: 10 -weeks post-surgery, a time when formalised rehabilitation is typically concluding, and at 26 weeks post-surgery, a time when recovery is typically plateauing $[3,6,7]$.

Further, our cohort characteristics signify an elderly population of people with end-stage osteoarthritis with significant impairment as indicated by the very low mean baseline Oxford scores (mean 17 from a maximum of 48), and the poor baseline walk tests which are well below the typical distances $(582 \mathrm{~m})$ measured in healthy 
70-year olds [11]. These characteristics, including their comorbidities, typify TKA populations captured locally $[3,4]$ as well as those captured internationally [6-8]. Our observations, therefore, should be both useful to clinicians involved in the rehabilitation of TKA recipients and be broadly generalizable.

By using both anchor-based and distribution-based approaches and then assessing the level of agreement between the thresholds obtained by each approach, we have identified a slight or more improvement threshold at 26-weeks post-surgery for the 6MWT in a TKA cohort. Based on triangulation of all four methods (two ROC approaches utilising patientperceived change, and two distributional approaches), and considering only the anchor-based and distributional thresholds with good agreement, it appears that the true threshold of a minimally important change is between $26 \mathrm{~m}$ and $55 \mathrm{~m}$. Interestingly, and probably importantly, the threshold range we have identified appears consistent with patient-perceived change thresholds for the 6MWT determined in other patient populations using anchor-based methodologies. For patients with heart disease, it has been estimated to be $25 \mathrm{~m}$ [18]. In older adults with mobility impairments, a small meaningful change has been found to be 19 to $22 \mathrm{~m}$ and a more substantial change has been found to be 47 to $49 \mathrm{~m}$ [19]. An MID of $25 \mathrm{~m}$ was identified for patients with COPD [24].

In determining the contribution this study makes to this area, our study has strengths and limitations. The strengths of our study lie in the comparatively large sample size, its prospective, longitudinal design, and the inclusion of participants from both arms of our combined randomised and observational study - the latter enhancing the generalisability of our findings. We also used multiple methods to establish the one threshold we did identify whilst considering the potential confounders of time and patient characteristics. Further, our study describes an improvement threshold in the 6MWT post-TKA that can be applied at the level of the individual. The use of the ROC curve approach allows the identification of important patient level-change, whereas approaches only applying distribution-based methodologies necessarily confine their changes to group-level change only [16, 36].

That we identified a range over which small improvement may be considered to have occurred as opposed to a single 'cut-off' figure is unusual when determining MIDs, but may be considered quite useful. This is because it allows flexibility in how we perceive improvement for the individual and within groups, acknowledging that there are multiple non-medical variables or life events that may influence a person's recovery post-TKA. Thus, there is not likely to be a single MID threshold that is universally representative. We also note that it is likely that future researchers in this area (arthroplasty, $6 \mathrm{MWT}$ and MID) will confront the same issue we faced with too few people reporting slight improvement, thus necessitating slight or more improvement thresholdtype categorisations. This is because for many if not all TKA cohorts, very large improvements in various outcomes, including mobility, are typically seen $[4,8,20]$.

Another difficult challenge in this area is applying a global question which captures all elements of improvement. Whilst we applied a global anchor which would allow us to compare our findings to others exploring change thresholds for the 6MWT, it may not have captured all the elements of improvement (or deterioration) in walking ability as perceived by the patient (and this would be the case for previous studies applying a similar anchor). Consequently, a lack of ability of our global question to capture all elements of improvement may have contributed to the weak correlations observed between the transition responses and measured improvements in walk distance. The $6 \mathrm{MWT}$ is essentially a test of gait speed; improvement may have occurred in other dimensions such as movement quality and, thus, not have been detected by the $6 \mathrm{MWT}$ or, for that matter, any other of the time-based mobility tests such as the timed up-and-go or $15 \mathrm{~m}$ walk test commonly used to test mobility after TKA [38]. It would appear a more specific question around improvement in speed per se or the use of a mobility test that is not time-based may be required to secure a greater correlation between measured change and perceived change, and, thus, achieve greater precision in a patient-perceived improvement threshold. Of course recall bias or response shift may also have contributed to the weak correlations observed, and this is not likely to be helped by a different global question. It should also be acknowledged that it is known that there is even poor concurrent validity between performance measures and what patients perceive they can do after TKA [20], thus, a more precise patient-perceived anchor or improvement for the 6MWT may never be found.

\section{Conclusions}

In conclusion, though the 6MWT is commonly used to evaluate recovery after TKA, uncertainty exists as to what is considered a minimal or even large improvement as perceived by the patient. Using multiple methods and subsequent triangulation of these methods, the likely minimum threshold about which patient-perceived improvement from pre-surgical status can be considered to have occurred is between 26 and $55 \mathrm{~m}$ at approximately six months after surgery. 


\section{Appendix}

Table 4 Growth curve analyses for the 6MWT

\begin{tabular}{llll}
\hline Parameter & Estimate & $95 \% \mathrm{Cl}$ & Sig \\
\hline Intercept & 490.0 & 398.2 to 581.8 & $<0.001$ \\
Magnitude of change & 73.8 & 50.2 to 97.3 & $<0.001$ \\
Rate of change over time & -20.5 & -32.0 to -9.0 & 0.001 \\
Gender & 62.4 & 39.0 to 85.8 & $<0.001$ \\
Age & -2.9 & -4.2 to -1.5 & $<0.001$
\end{tabular}

Post-operative average distance of $6 \mathrm{MWT}$ at 10 -weeks post-surgery can be calculated using the following equations where males are coded " 0 " and females are "1"

Males: $490+(1 \times 73.8)+(1 \times-20.5)+(0 \times 62.4)+(66.2 \times-2.9)$, where 66.2 is the average age

Females: $490.0+(1 \times 73.8)+(1 x-20.5)+(1 \times 62.4)+(66.8 x-2.9)$, where 66.8 is

the average age

\section{Abbreviations}

6MWT: 6-min walk test; AUC: Area under the curve; MID: Minimal important difference; ROC: Receiver operating characteristic; SEM: Standardised error or measurement; TKA: Total knee arthroplasty

\section{Acknowledgements}

We acknowledge all our patient participants. We also acknowledge Sarah-Jane Lucas, Jason Li, and Minh Nguyen for their assistance with data collection.

\section{Role of funding}

The study was funded in part by the HCF Health Research Foundation.

\section{Availability of data and materials}

Data will be made available to individuals on request.

\section{Authors' contributions}

JN conceived the study; JN and MB designed the study; KM performed the statistical analysis; MB, RF, RW collected the data; JN, KM and MB prepared the first draft of the manuscript; All authors read and contributed to the final manuscript; JN is responsible for the integrity of the work and is the corresponding author.

\section{Competing interests}

The authors declare that they have no competing interests.

\section{Consent for publication}

Not applicable.

\section{Ethics approval and consent to participate}

The study was approved as part of the HIHO study [2] by the St Vincent's Hospital HREC (Approval number 11/125). All participants provided written, informed consent.

\section{Author details}

${ }^{1}$ Whitlam Orthopaedic Research Centre, Orthopaedic Department, Liverpool Hospital, Locked Bag 7103, Liverpool BC, 1871 Sydney, NSW, Australia. ${ }^{2}$ South West Sydney Clinical School, UNSW, Sydney, Australia. ${ }^{3}$ Ingham Institute of Applied Medical Health Research, Sydney, Australia. ${ }^{4}$ Faculty of Medicine and Health Sciences, Macquarie University, Sydney, Australia. ${ }^{5}$ Braeside Hospital, Hammondcare Group, Sydney, Australia. ${ }^{6}$ Physiotherapy Department, Campbelltown Hospital, Sydney, Australia. ${ }^{7}$ Occupational Therapy Department, Fairfield Hospital, Sydney, Australia.

Received: 21 April 2016 Accepted: 9 September 2016 Published online: 13 September 2016

\section{References}

1. Naylor JM, Crosbie J, Ko V. Is there a role for rehabilitation streaming following TKA? Preliminary insights from a randomised controlled trial. J Rehab Med. 2015;47:235-41.
2. Buhagiar MA, Naylor JM, Harris IA, Xuan W, Kohler F, Wright R. Hospital Inpatient versus $\mathrm{HOme}$-based rehabilitation after knee arthroplasty (The HIHO study): study protocol for a randomized controlled trial. Trials. 2013;14:432.

3. Ko V, Naylor J, Harris I, Crosbie J, Yeo A, Mittal R. One-to-one therapy is not superior to group or home-based therapy after total knee arthroplasty: A randomized, superiority trial. J Bone Joint Surgy - Series A. 2013:95:1942-9.

4. Harmer AR, Naylor JM, Crosbie J, Russell T. Land-based versus water-based rehabilitation following total knee replacement: A randomized, single-blind trial. Arthritis Rheum. 2009;61:184-91.

5. Dobson F, Hinman RS, Roos EM, Abbott JH, Stratford P, Davis AM, et al. OARSI recommended performance-based tests to assess physical function in people diagnosed with hip or knee osteoarthritis. Osteoarthritis Cartilage. 2013;21(8):1042-52.

6. Moffet H, Collet JP, Shapiro SH, Paradis G, Marquis F, Roy L. Effectiveness of intensive rehabilitation on functional ability and quality of life after first total knee arthroplasty: a single-blind randomized controlled trial. Arch Phys Med Rehabil. 2004;85:546-56.

7. Kramer JF, Speechley M, Bourne R, Rorabeck C, Vaz M. Comparison of clinicand home-based rehabilitation programs after total knee arthroplasty. Clin Orthop Relat Res. 2003:410:225-34.

8. Kennedy DM, Stratford PW, Wessel J, Gollish JD, Penney D. Assessing stability and change of four performance measures: a longitudinal study evaluating outcome following total hip and knee arthroplasty. BMC Musculoskelet Disord. 2005;6:3

9. Statement ATS. Guidelines for the Six-Minute Walk Test. Am J Respir Crit Care Med. 2002;166:111-7

10. Yoshida Y, Mizner RL, Ramsey DK, Snyder-Mackler L. Examining outcomes from total knee arthroplasty and the relationship between quadriceps strength and knee function over time. Clin Biomech (Bristol, Avon). 2008;23: 320-8.

11. Ko V, Naylor JM, Harris IA, Crosbie J, Yeo AET. The six-minute walk test is an excellent predictor of functional ambulation after total knee arthroplasty. BMC Musculoskelet Disord. 2013:14:145.

12. Jakobsen $T L$, Kehlet $H$, Bandholm T. Reliability of the 6-min walk test after total knee arthroplasty. Knee Surg Sports Traumatol Arthrosc. 2013;21:2625-8.

13. Naylor JM, Hayen A, Davidson E, Hackett D, Harris IA, Kamalasena G, Mittal R Minimal detectable change for mobility and patient-reported tools in people with osteoarthritis awaiting arthroplasty. BMC Musculoskelet Disord. 2014;15:235.

14. Revicki D, Hays RD, Cella D, Sloan J. Recommended methods for determining responsiveness and minimally important differences for patient-reported outcomes. J Clin Epi. 2008;61:102-9.

15. Osteoarthritis Chronic Care Program. https://www.aci.health.nsw.gov.au/ resources/musculoskeletal/osteoarthritis_chronic_care_program/ osteoarthritis-chronic-care-program. Accessed 10 Sept 2016.

16. Mills KA, Naylor JM, Eyles JP, Roos EM, Hunter DJ. Examining the minimal important difference of patient-reported outcome measures for individuals with knee osteoarthritis: A model using the Knee Injury and Osteoarthritis Outcome Score. J Rheumatol. 2016;43:395-404.

17. Puhan MA, Mador MJ, Held U, Goldstein R, Guyatt GH, Schunemann HJ. Interpretation of treatment changes in 6-minute walk distance in patients with COPD. Eur Respir J. 2008:32:637-43.

18. Gremeaux V, Troisgros O, Benaïm S, Hannequin A, Laurent Y, Casillas JM, et al. Determining the minimal clinically important difference for the sixminute walk test and the 200-meter fast-walk test during cardiac rehabilitation program in coronary artery disease patients after acute coronary syndrome. Arch Phys Med Rehabil. 2011;92:611-9.

19. Perera S, Mody SH, Woodman RC, Studenski SA. Meaningful change and responsiveness in common physical performance measures in older adults. J Am Geriatr Soc. 2006;54:743-9.

20. Mizner RL, Petterson SC, Clements KE, Zeni JA, Irrgang J, Snyder-Mackler L. Measuring functional improvement after total knee arthroplasty requires both performance-based and patient-report assessments: a longitudinal analysis of outcomes. J Arthroplasty. 2011;26:728-37.

21. McLeod LD, Coon CD, Martin SA, Fehnel SE, Hays RD. Interpreting patientreported outcome results: US FDA guidance and emerging methods. Expert Rev Pharmacoecon Outcomes Res. 2011:11:163-9.

22. McPhail S, Haines T. Response shift, recall bias and their effect on measuring change in health-related quality of life amongst older hospital patients. Health Qual Life Outcomes. 2010;8:65. 
23. Jaeschke R, Singer J, Guyatt GH. Measurement of health status: Ascertaining the minimal clinically important difference. Control Clin Trials. 1989;10:407-15.

24. Holland AE, Hill CJ, Rasekaba T, Lee A, Naughton MT, McDonald CF. Updating the minimal important difference for six-minute walk distance in patients with chronic obstructive pulmonary disease. Arch Phys Med Rehabil. 2010;91:221-5.

25. Farrar JT, Dworkin RH, Max MB. Use of the cumulative proportion of responders analysis graph to present pain data over a range of cutoff points: making clinical trial data more understandable. J Pain Symptom Manage. 2006;31:369-77.

26. Shek DTL, Ma CMS. Longitudinal data analyses using linear mixed models in SPSS: concepts, procedures and illustrations. Sci World J. 2011;11:42-76.

27. Field A. Discovering Statistics Using IBM SPSS Statistics. 4th ed. London: Sage Publications Ltd; 2013. Chapter 20.

28. Brozek JL, Guyatt GH, Schunemann HJ. How a well-grounded minimal important difference can enhance transparency of labelling claims and improve interpretation of a patient-reported outcome measure. Health Qual Life Outcomes. 2006;4:69.

29. Davis AM, Perruccio AV, Lohmander LS. Minimally clinically important improvement: all non-responders are not really non-responders an illustration from total knee replacement. Osteoarthritis Cartilage. 2012;20:364-7.

30. Turner D, Schunemann HJ, Griffith LE, Beaton DE, Griffiths AM, Critch JN, et al. Using the entire cohort in the receiver operating characteristic analysis maximizes the precision of the minimal important difference. J Clin Epi. 2009;62:374-9.

31. Fan J, Upadhyve S, Worster A. Understanding receiver operating characteristic (ROC) curves. Can J Emerg Med. 2006;8:19-20.

32. de Vet HCW, Terluin B, Knol DL, Roorda LD, Mokkink LB, Ostelo RWJG, et al. Three ways to quantify uncertainty in individually applied 'minimally important change' values. J Clin Epidemiol. 2010;63:37-45.

33. Aletaha D, Funovits J, Ward MM, Smolen JS, Kvien TK. Perception of improvement in patients with rheumatoid arthritis varies with disease activity levels at baseline. Arthritis Rheum. 2009;61:313-20.

34. Kazis LE, Anderson JJ, Meenan RF. Effect sizes for interpreting changes in health status. Med Care. 1989;27(supplement):S178-89.

35. Norman GR, Sloan JA, Wyrwich KW. Interpretation of changes in healthrelated quality of life: the remarkable universality of half a standard deviation. Med Care. 2003;41:582-92.

36. King MT. A point of minimal important difference (MID): a critique of terminology and methods. Expert Rev Pharmacoeconomics Outcomes Res. 2011;11:171-84.

37. Tooth LR, Ottenbacher KJ. The kappa statistic in rehabilitation research: an examination. Arch Phys Med Rehabil. 2004;85:1371-6.

38. Dobson F, Hinman RS, Hall M, Terwee CB, Roos EM, Bennell K. Measurement properties of performance-based measures to assess physical function in hip and knee osteoarthritis: a systematic review. Osteoarthritis Cartilage. 2012;20:1548-62.

\section{Submit your next manuscript to BioMed Central and we will help you at every step:}

- We accept pre-submission inquiries

- Our selector tool helps you to find the most relevant journal

- We provide round the clock customer support

- Convenient online submission

- Thorough peer review

- Inclusion in PubMed and all major indexing services

- Maximum visibility for your research

Submit your manuscript at www.biomedcentral.com/submit

) Biomed Central 